\title{
DISTRIBUIÇÃO TEMPORAL DOS CASOS E DA MORTALIDADE INFANTIL POR SÍFILIS CONGÊNITA NAS CINCO REGIÕES GEOGRÁFICAS DO BRASIL ENTRE 2009 E 2018
}

\section{TEMPORAL DISTRIBUTION OF CASES AND OF INFANT MORTALITY FROM CONGENITAL SYPHILIS IN THE FIVE GEOGRAPHICAL REGIONS OF BRAZIL BETWEEN 2009 AND 2018}

\author{
Fernando Maia Coutinho' ${ }^{1}$, Yuri Fadi Geha1 ${ }^{1}$, Luzivan Costa Reis², \\ Wesley Luciano Kaizer ${ }^{3}$, Tiago Arantes ${ }^{4}$, Carolina Bastos Brega ${ }^{5}$
}

\section{RESUMO}

Introdução: No mundo, estima-se que cerca de 1,8 milhões de gestantes estejam infectadas por sífilis, apesar de apenas $10 \%$ desse valor sejam diagnosticados e devidamente tratados. Nesse sentido, o presente estudo tem por objetivo descrever a evolução temporal da incidência dos casos e das taxas de mortalidade por sífilis congênita (SC) em menores de 1 ano, bem como comparar o impacto dessa mortalidade por regiões do Brasil, entre 2009 e 2018.

Métodos: Trata-se de um estudo ecológico, retrospectivo, com dados do Sistema de Informação e Agravos de Notificação (SINAN), Sistema de Informações sobre Mortalidade (SIM) e do Sistema de Informação sobre Nascidos Vivos (SINASC).

Resultados: A incidência de SC em indivíduos menores de 1 ano apresentou um acréscimo de $328,57 \%$ nas taxas notificadas entre $2009(2,1 / 1.000)$ e $2018(9 / 1.000)$. A média anual de incidência $\left(\beta_{1}\right)$ por SC foi de 0,80 (IC95\% 0,75-0,86; $\left.p=00006\right)$, com um coeficiente de determinação $\left(R^{2}\right)$ igual a $0,99 \%$. A variação média da taxa de mortalidade por SC $\left(\beta_{1}\right)$ foi 0,006/1.000 (IC95\% 0,005-0,008; $p=0,00004, R^{2}=0,93 \%$ ). A região Sudeste apresentou a maior proporção de mortalidade pela doença entre menores de 1 ano por SC, com 43,14\% dos óbitos.

Conclusão: Foi observada uma correlação positiva da incidência e da mortalidade por SC entre menores de 1 ano no Brasil ao longo dos anos analisados, sendo perceptível elevadas taxas entre as cincos regiões geográficas, sugerindo a necessidade de maior atenção à triagem no pré-natal e melhorias na capacitação dos serviços de saúde.

Palavras-chaves: Sífilis congênita; Mortalidade infantil; Distribuição temporal

\section{ABSTRACT}

Introduction: Worldwide, it is estimated that about 1.8 million pregnant women are infected with syphilis, although only $10 \%$ of them are diagnosed and treated appropriately. Therefore, the present study aims to describe the temporal evolution of the incidence of cases of and mortality rates from congenital syphilis (CS) in children younger than 1 year, as well as to compare the impact of this mortality by regions of Brazil between 2009 and 2018.

Methods: This is an ecological, retrospective study, with data from the Information System and Notifiable Diseases (SINAN), Mortality Information System (SIM) and the Information System on Live Births (SINASC).

Results: The incidence of CS in individuals younger than 1 year showed an increase of $328.57 \%$ in the rates recorded between $2009(2.1 / 1,000)$ and $2018(9 / 1,000)$. The mean
Clin Biomed Res. 2021;41(4):1-8

1 Faculdade de Medicina, Universidade Federal do Pará. Belém, PA, Brasil.

2 Programa de Pós-Graduação em Genética e Biologia Molecular, Universidade Federal do Rio Grande do Sul. Porto Alegre, RS, Brasil.

3 Departamento de Informática, Universidade Federal do Rio Grande do Sul. Porto Alegre, RS, Brasil.

4 Centro de Ciências Biológicas, Universidade Estadual de Londrina. Londrina, PR, Brasil.

5 Faculdade de Medicina, Centro Universitário do Pará. Belém, PA, Brasil.

Autor correspondente: Yuri Fadi Geha yuri.geha@ics.ufpa.br Faculdade de Medicina, Universidade Federal do Pará Rua Augusto Corrêa, 01 66075-110, Belém, PA, Brasil. 
Coutinho et al.

annual incidence $(\beta 1)$ per CS was 0.80 (95\% confidence interval [95\%Cl] 0.75-0.86; $p=00006)$, with a coefficient of determination (R2) equal to $0.99 \%$. The mean variation in mortality rate from SC ( $\beta 1)$ was $0.006 / 1,000(95 \% \mathrm{Cl} 0.005-$ $0.008 ; p=0.00004, \mathrm{R} 2=0.93 \%)$. The southeast region had the highest proportion of mortality from the disease among children younger than 1 year due to CS, with $43.14 \%$ of deaths.

Conclusion: There was a positive correlation between incidence of and mortality from CS among children younger than 1 year in Brazil over the years analyzed, with high rates in the five geographic regions, suggesting the need for greater attention to prenatal screening and improvements in the training of health service professionals.

Keywords: Congenital syphilis; Infant mortality; Temporal distribution

\section{INTRODUÇÃO}

A sífilis é ocasionada por uma bactéria denominada de Treponema Pallidum, pertencente ao gênero Treponema e à família Treponemataceae, sendo a espécie Treponema pallidum, subespécie pallidum, responsável por sua patologia ${ }^{1}$. A transmissão deste patógeno pode ocorrer tanto por uma via horizontal (relações sexuais), a partir do contato com lesões características contagiantes, como a úlcera genital indolor (cancro duro), quanto por via vertical (sífilis congênita), existindo, entretanto, a possibilidade de transmissão em meio a vias indiretas (objetos perfurantes, cortantes e transfusão sanguínea) $)^{1,2}$.

No mundo, a sífilis apresenta-se de forma reemergente em países desenvolvidos e em elevada incidência nas regiões da América Latina e África ${ }^{3-5}$, com estudos indicando que cerca de 1,8 milhões de gestantes no mundo estejam infectadas, embora apenas $10 \%$ desse valor seja diagnosticado e devidamente tratado . $^{\circ}$ Assim, diante de tais indicadores, a Organização Mundial de Saúde (OMS) estabeleceu estratégias para o enfrentamento da sífilis congênita (SC), atuando de forma reduzir os índices subjacentes à transmissão vertical ${ }^{7}$. Tal iniciativa, associada à Organização Pan-Americana de Saúde (OPAS), estabeleceu como meta, até 2015, alcançar a taxa de incidência de $0,5 / 1.0$ para cada 1.000 nascidos vivos (NV). A meta adotada é a mesma estabelecida pelos Ministérios de Saúde da região, como o Brasil, apesar das formas congênita e adquiridas em gestantes já apresentarem-se como forma de notificação compulsória desde 1986 e 2005, respectivamente ${ }^{8-11}$.

A SC é considerada um indicador de qualidade da assistência pré-natal de uma determinada população, devido à existência de protocolos, triagem sorológica e tratamento de gestantes e seus respectivos parceiros no Sistema Único de Saúde (SUS), possibilitando que tal patologia seja evitável em condições ideais ${ }^{12,13}$. Contudo, apesar de ser um evento sentinela, a subnotificação de casos no Brasil é presente e alarmante ${ }^{14}$, apresentando, entre 1998 e 2012, um quantitativo superior a 80.000 mil casos de SC em indivíduos menores de 1 ano, o que representa, estatisticamente, uma taxa de 3,3/1.000 NV em 2011, valor seis vezes superior ao determinado pela OPAS ${ }^{6,15}$.
Estima-se que cerca de $40 \%$ dos casos de transmissão congênita estejam relacionados a três desfechos principais: óbito perinatal (óbito fetal-tempo de gestação maior ou igual a 22 semanas + óbito neonatal - tempo de vida de 0 a 6 dias), natimorto e aborto espontâneo ${ }^{16}$. Dessa forma, a inclusão da sífilis como Infecção Sexualmente Transmissível (IST) de notificação compulsória representa uma importante medida da vigilância em saúde para a contenção dos impactos ocasionados tanto pela sífilis em gestantes quanto pela SC, transmitida seja por via transplacentária ou de forma direta pelo parto.

Desse modo, considerando os impactos ocasionados pelos casos de SC para o sistema de saúde do país e a necessidade de dados quantitativos para o planejamento e implementação de medidas de controle por agentes de saúde, o presente estudo tem por objetivo descrever a evolução temporal da incidência dos casos e das taxas de mortalidade por sífilis congênita (SC) em menores de 1 ano, bem como comparar o impacto dessa mortalidade por regiões do Brasil, entre 2009 e 2018.

\section{MÉTODOS}

Trata-se de um estudo ecológico, retrospectivo, referente à incidência e às taxas de mortalidade ocorridos por SC entre menores de 1 ano de idade no Brasil, entre o período de 2009 a 2018. Os dados analisados são de casos confirmados e registrados no Sistema de Informação e Agravos de Notificação (SINAN), no Sistema de Informações sobre Mortalidade (SIM) e no Sistema de Informação sobre Nascidos Vivos (SINASC). As ferramentas utilizadas para a verificação dos registros estão disponíveis em: http://www2.datasus.gov.br/.

Posteriormente à consecução dos dados, referentes aos gráficos e tabelas, estes foram analisados e tabulados no programa Microsoft Excel ${ }^{\circ}$, com auxílio da plataforma TABWIN-DATASUS, programa disponibilizado pelo Ministério da Saúde. Disponível: www.datasus.gov.br

A incidência de SC em crianças menores de 1 ano foi calculada a partir da razão entre o número de novos casos da doença, detectados anualmente, sobre o número de NV, sendo o quociente da 
operação multiplicado por 100. A taxa de mortalidade foi calculada, para cada ano, utilizando o número de óbitos registrados pelo SIM como numerador e a população de NV, somada aos óbitos de menores de 1 ano, como denominador, sendo o quociente dessa operação multiplicado por 1.000 .
Abaixo, a Tabela 1 e 2 dispõe, respectivamente, a estimativa do número absoluto de $\mathrm{NV}$, de acordo com os dados disponíveis no SINASC, e o número de casos confirmados de SC, segundo dados do SINAN, em crianças menores de 1 ano de idade, entre os anos de análise do estudo e as regiões geográficas do Brasil.

Tabela 1: Estimativa populacional do número de nascidos vivos (NV), segundo período de análise, 2009 a 2018.

\begin{tabular}{ccccccc}
\hline $\begin{array}{c}\text { Ano do } \\
\text { nascimento }\end{array}$ & $\begin{array}{c}\text { Região } \\
\text { Norte }\end{array}$ & $\begin{array}{c}\text { Região } \\
\text { Nordeste }\end{array}$ & $\begin{array}{c}\text { Região } \\
\text { Sudeste }\end{array}$ & Região Sul & $\begin{array}{c}\text { Região Centro- } \\
\text { Oeste }\end{array}$ & Total \\
\hline 2009 & 310726 & 865098 & 1119231 & 366358 & 220168 & 2881581 \\
2010 & 306422 & 841160 & 1123593 & 369905 & 220788 & 2861868 \\
2011 & 313745 & 851004 & 1143741 & 378093 & 226577 & 2913160 \\
2012 & 308375 & 832631 & 1152846 & 381658 & 230279 & 2905789 \\
2013 & 313272 & 821458 & 1147627 & 386983 & 234687 & 2904027 \\
2014 & 321682 & 833090 & 1182949 & 396462 & 245076 & 2979259 \\
2015 & 320924 & 846374 & 1196232 & 406529 & 247609 & 3017668 \\
2016 & 307526 & 796119 & 1127499 & 391790 & 234866 & 2857800 \\
2017 & 312682 & 817311 & 1151832 & 397604 & 244106 & 2923535 \\
2018 & 319228 & 836850 & 1147006 & 395857 & 245991 & 2944932 \\
Total & 3134582 & 8341095 & 11492556 & 3871239 & 2350147 & 29189619 \\
\hline
\end{tabular}

Tabela 2: Número de casos confirmados de sífilis congênita entre crianças menores de 1 ano por regiões geográficas, segundo período de análise, 2009 a 2018.

\begin{tabular}{ccccccc}
\hline $\begin{array}{c}\text { Ano do } \\
\text { Diagnóstico }\end{array}$ & $\begin{array}{c}\text { Região } \\
\text { Norte }\end{array}$ & $\begin{array}{c}\text { Região } \\
\text { Nordeste }\end{array}$ & $\begin{array}{c}\text { Região } \\
\text { Sudeste }\end{array}$ & Região Sul & $\begin{array}{c}\text { Região Centro- } \\
\text { Oeste }\end{array}$ & Total \\
\hline 2009 & 764 & 2058 & 1809 & 540 & 352 & 5523 \\
2010 & 633 & 2300 & 2989 & 655 & 344 & 6921 \\
2011 & 792 & 3223 & 4121 & 923 & 399 & 9458 \\
2012 & 1065 & 4256 & 5961 & 1323 & 644 & 13249 \\
2013 & 1297 & 5063 & 7019 & 1764 & 902 & 16045 \\
2014 & 1236 & 5167 & 7287 & 1921 & 968 & 16579 \\
2015 & 1432 & 6150 & 8445 & 2807 & 1137 & 19971 \\
2016 & 1751 & 6061 & 9185 & 3159 & 1249 & 21405 \\
2017 & 2203 & 7057 & 10340 & 3600 & 1433 & 24633 \\
2018 & 2029 & 7010 & 9732 & 3303 & 1376 & 23450 \\
Total & 13212 & 48395 & 67007 & 20059 & 8812 & 157485 \\
\hline
\end{tabular}


A análise da incidência e da mortalidade por SC foi estabelecida a partir de uma regressão linear simples, por meio da seguinte fórmula:

$$
\mathrm{Y}_{\mathrm{i}}=\beta_{0}+\beta_{1} \mathrm{X}_{\mathrm{i}}+\mu \mathrm{i}
$$

Os termos que compõem a expressão possuem os seguintes significados: Yi é o coeficiente do parâmetro de análise (incidência e mortalidade por SC entre menores de 1 ano) como variável dependente; $\beta_{0}$ expressa a constante do modelo; $\beta 1$ refere-se à variação média anual do coeficiente; $X$ representa os anos transcorridos como uma variável independente; $\mu$ i representa o erro aleatório. $\mathrm{O}$ ajuste dos modelos se deu pelo coeficiente de determinação $\left(R^{2}\right)$ que mede a proporção de variação anual e a significância do modelo ( $p$-valor para o modelo).

ANOVA (Teste F) foi utilizado para comparar a taxa de mortalidade infantil (menores de 1 ano) por SC nas cinco regiões geográficas (Norte, Nordeste, Sul, Sudeste, Centro-Oeste), entre 2009 a 2018. O teste post hoc de Bonferroni's foi aplicado para analisar pares específicos de amostras para dominância estocástica. Foram calculados os coeficientes de correlação de Spearman e Pearson ( $\rho$ ) entre o número de regiões geográficas e a taxa de mortalidade infantil por SC em crianças menores de 1 ano. Foram consideradas estatisticamente significativas as correlações com valor de $p<0,05$. Todas as análises foram realizadas pelo Software R, versão 3.6.0 (https://www.r-project.org/).
Considerando que este estudo utiliza a disponibilidade de dados públicos secundários, não foram requeridos os Termos de Consentimentos Livres e Esclarecidos (TCLE), pois não há existência de identificação individual. Os dados aqui incluídos referem-se a casos de SC em indivíduos identificados no sistema DATASUS. Todos os indivíduos humanos possuíam registros anônimos nos sites do SINAN/ SIM/SINASC. Por tratar-se de um banco de dados obtido em uma base pública, de acesso gratuito (Conselho Nacional de Saúde 2012), a Resolução 466/12 da legislação brasileira não exige a aprovação do Comitê de Ética em Pesquisa.

\section{RESULTADOS}

Durante os anos analisados, observou-se um aumento consecutivo da taxa de incidência de $\mathrm{SC}$ em indivíduos menores de 1 ano de idade, representado por um acréscimo percentual de $328,57 \%$ das taxas notificadas entre os anos de 2009 (2,1/1.000 NV) e $2018(9 / 1.000 \mathrm{NV})$. A média de crescimento anual da taxa $(\beta 1)$ observada no período foi de 0,80 (IC95\% $0,75-0,86 ; p=00006)$, com um coeficiente de determinação $\left(R^{2}\right)$ igual a $0,99 \%$ (Figura 1). Podese observar, também, que o maior aumento na taxa de incidência ocorreu entre os anos de 2016 $(7,40 / 1.000 \mathrm{NV})$ e 2017 (8,5/1.000 NV).

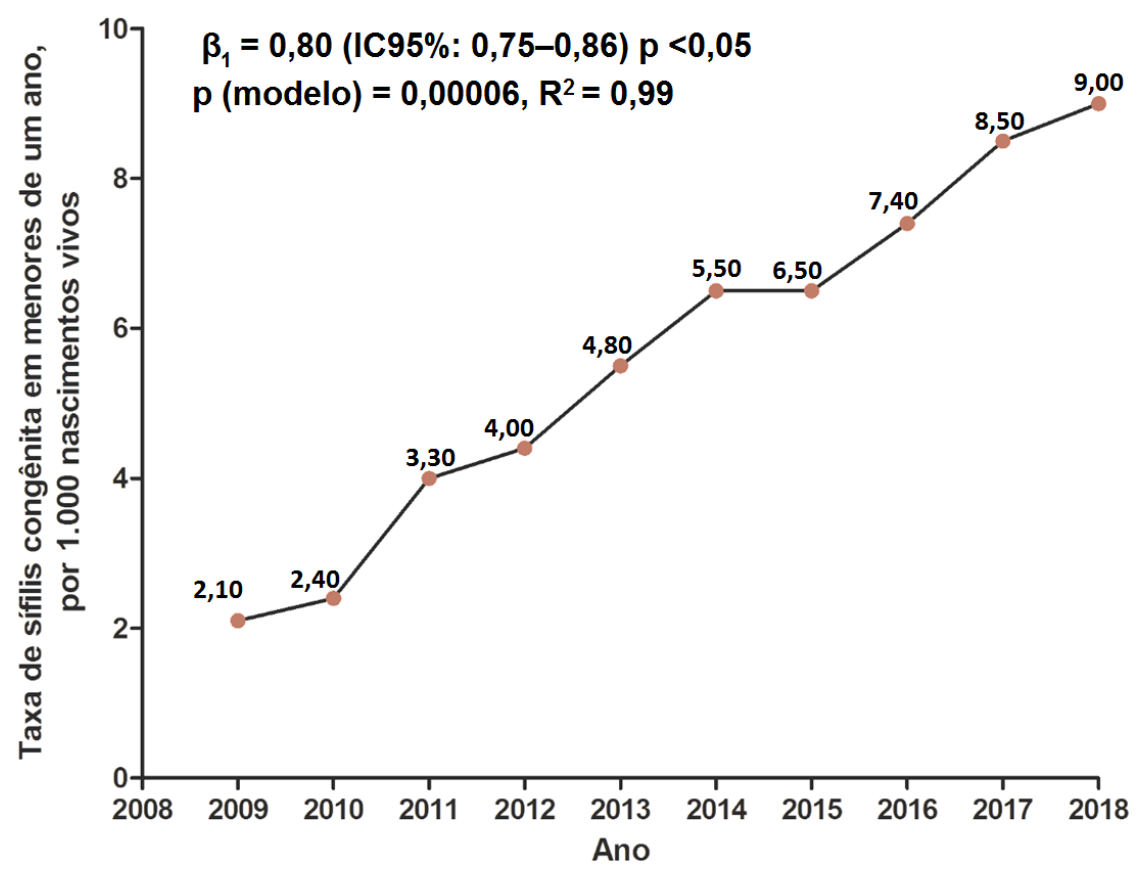

Figura 1: Incidência de SC em indivíduos com idade inferior a 1 ano de idade no Brasil $(\mathrm{n}=1662)$, segundo período de análise, 2009 a 2018. 
Quanto à taxa de mortalidade da doença em menores de 1 ano, evidenciou-se um crescimento consecutivo entre os anos analisados, percentualmente, correspondente a um aumento de $300 \%$, com 0,002 óbitos/ 1.000 NV em 2009 e $0,08 / 1.000 \mathrm{NV}$ em 2018. A variação média da taxa de mortalidade $\left(\beta_{1}\right)$ foi equivalente a $0,06 / 1.000 \mathrm{NV}$
(IC95\% 0,005-0,008; p=0,00004), representada por um coeficiente de determinação $\left(R^{2}\right)$ de $0,93 \%$. Houve, ainda, a apresentação de taxas crescentes até o ano de 2015, seguida por uma queda de 0,01/1.000 NV no ano de 2016, retomando, posteriormente, o coeficiente de 0,08/1.000 NV até o final do estudo (Figura 2).

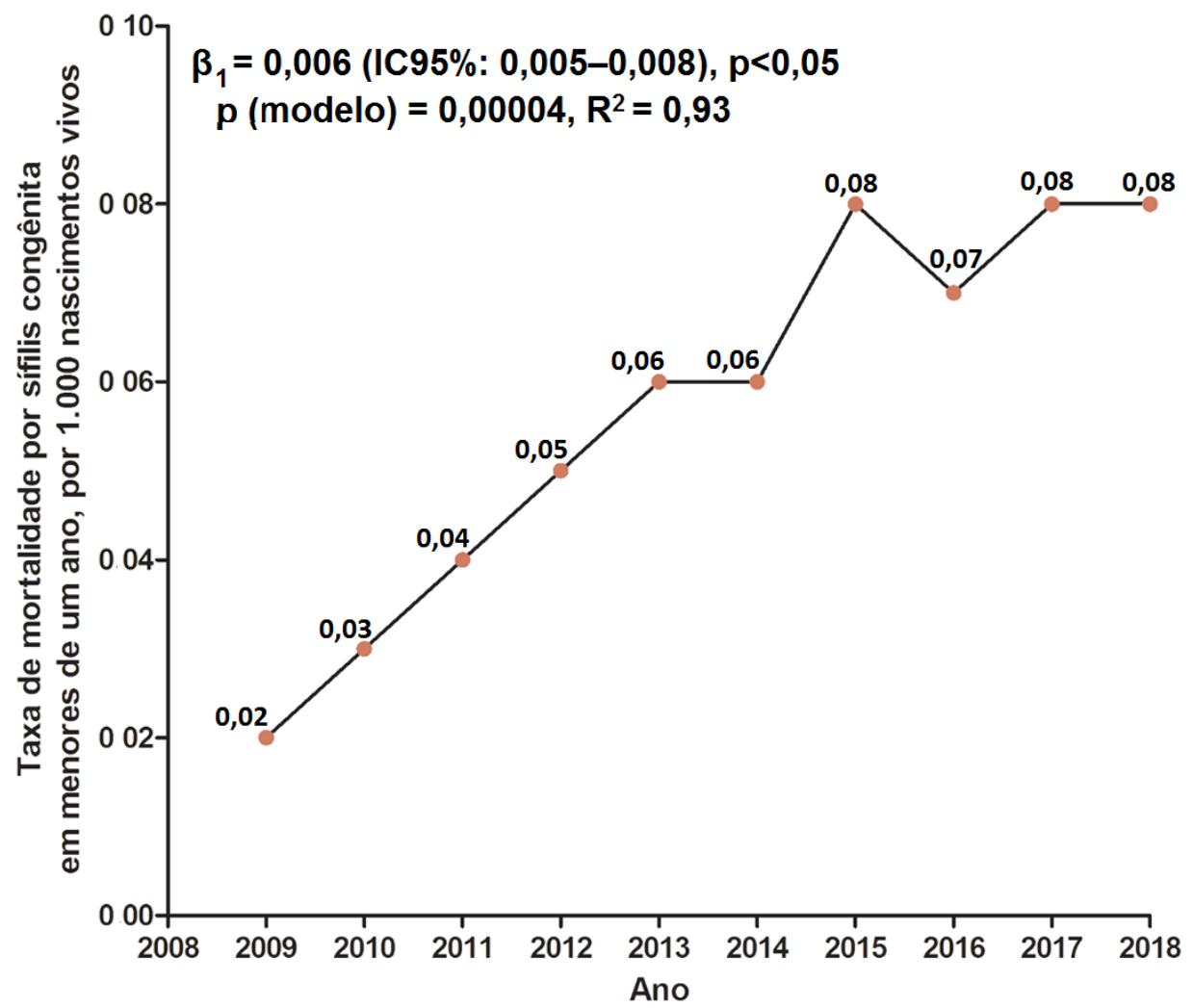

Figura 2: Taxa de mortalidade por SC em indivíduos com idade inferior a 1 ano de idade no Brasil, segundo período de análise, 2009 a 2018.

Entre as regiões brasileiras, foi observado que a região Sudeste apresentou a maior proporção de mortalidade entre menores de 1 ano por SC, com $43,14 \%$ dos óbitos notificados pela doença, seguida da região Nordeste $(29,66 \%$,$) , Norte (12,15 \%)$, Sul $(9,69 \%)$ e Centro-Oeste $(5,35 \%)$; Teste $F=47,35$;
$R^{2}=0,84 ; p<0,0001$. Na Figura 3, observa-se que tanto as correlações de Pearson como de Spearman foram estatisticamente significativas para quase todas as regiões, sendo identificado o mesmo coeficiente de determinação $\left(R^{2}=0,88 \% ; 0,54 \%-0,88 \% ; p<0,0001\right)$ entre as regiões Nordeste e Centro-Oeste (Figura 3 ). 


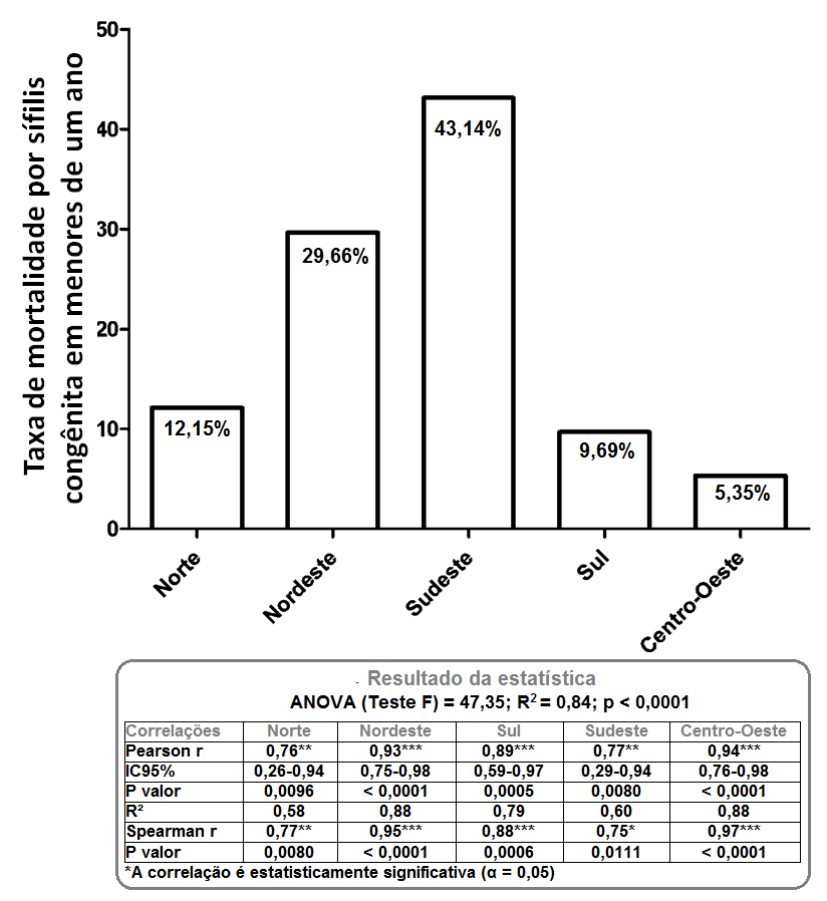

Figura 3: Taxa de mortalidade por SC em menores de 1 ano e correlações entre as regiões do Brasil, 2009 a 2018.

Por fim, foi realizada a análise dos dados por pares de regiões, sendo observada uma diferença estatisticamente significativa entre todos os pares de regiões formados, à exceção dos pares Norte versus Sul (teste de Bonferroni's $=0,75$ ), Norte versus CentroOeste (teste de Bonferroni's $=2,08$ ) e Sul versus
Centro-Oeste (teste de Bonferroni's = 1,32). A maior diferença de média encontrada foi entre Sudeste versus Centro-Oeste (valor do teste de Bonferroni's $=11,56$; $p<0,05)$. As diferenças completas de pares nas taxas de mortalidade por SC entre as cinco regiões brasileiras são apresentadas na Figura 3 e Tabela 3.

Tabela 3: Comparações entre pares de regiões e mortalidade por SC em menores de 1 ano, 2009 a 2018.

\begin{tabular}{lccc}
\hline \multicolumn{1}{c}{ Teste de Bonferroni's } & Diferença/média & Valor do Teste $^{1}$ & Resumo $^{1}$ \\
\hline Norte versus Nordeste & $-29,10$ & 5,35 & $* * *$ \\
Norte versus Sudeste & $-51,50$ & 9,48 & $* * *$ \\
Norte versus Sul & 4,100 & 0,75 & Não \\
Norte versus Centro-Oeste & 11,30 & 2,08 & Não \\
Nordeste versus Sudeste & $-22,40$ & 4,12 & $* *$ \\
Nordeste versus Sul & 33,20 & 6,11 & $* * *$ \\
Nordeste versus Centro-Oeste & 40,40 & 7,43 & $* * *$ \\
Sudeste versus Sul & 55,60 & 10,24 & $* * *$ \\
Sudeste versus Centro-Oeste & 62,80 & 11,56 & $* * *$ \\
Sul versus Centro-Oeste & 7,200 & 1,32 & Não \\
\hline
\end{tabular}

${ }^{1}$ Significância $(p<0,05)$. * Estatisticamente significativo.

\section{DISCUSSÃO}

A partir da análise dos dados coletados, os valores crescentes ao longo do período analisado quanto à taxa de detecção de sífilis em crianças menores de 1 ano, com um aumento percentual de percentual de $328,57 \%$, alcançando um valor de 9,00/1.000 NV, revelam índices alarmantes da incidência do cenário de sífilis infantil no país. Tal achado demonstra consonância com o levantamento epidemiológico de 1998 a 2014 realizado pelo Sistema de Informação de Agravos de Notificação (SINAN), com um registro 
de 104.853 casos de SC em indivíduos menores de 1 ano de idade em território nacional, especialmente na região Sudeste, com 48.015 casos $(45,8 \%)$, seguido pela região Nordeste com 32.884 casos $(31,4 \%)^{17}$.

Assim, o cenário nacional, especialmente nas regiões Nordeste, Norte e Centro-Oeste, que, historicamente, apresentavam os menores valores nacionais de incidência relacionados a essa patologia, devido à baixa testagem ${ }^{18}$, destacam um aumento da notificação dos casos de SC. Sendo esse o resultado de um possível reflexo da melhoria na notificação para essa infecção ao longo do tempo, assim como de avanços na vigilância epidemiológica e de um maior acesso de gestantes ao serviço de pré-natal existente nas Estratégias de Saúde da Família (ESF's) ${ }^{19}$.

$\mathrm{Em}$ relação à incidência, essa tendência de crescimento de casos notificados vem ocorrendo de forma global, como descrito nos achados de Read, Cristopher e Chow para 31 países $^{20}$. Tal cenário ocorre principalmente em países de alta renda, demonstrando que a transmissão considerável de casos de SC não é característica dos países com menores índices socioeconômicos, evidenciando a complexidade para a implementação de medidas efetivas para o combate a essa infecção.

Todavia, destaca-se que, embora os índices elevados, tais valores provavelmente encontram-se subnotificados em seus sistemas de registros, pois existem discrepâncias nos valores demonstrados em cada banco de dados. Por exemplo, de acordo com o Boletim Epidemiológico, no ano de 2007, foram notificados 126 casos de sífilis congênita em menores de um ano, todavia, o SINAN demonstrou 130 casos. Enquanto no ano de 2010, o Boletim Epidemiológico apresentou 201 casos e o SINAN apenas 44 registros, evidenciando episódios de inconsistência das informações ${ }^{14}$.

Vale ressaltar ainda que a cobertura de assistência ao pré-natal no Brasil é extremamente ampla, possuindo um caráter universal e valores superiores a $90 \%$ em muitas regiões do país, apesar da existência de regiões com valores menores, como a região Norte, que apresenta características geográficas e culturais específicas ${ }^{18}$. O processo considerado essencial para o sucesso dessa cobertura assistencial, segundo a $\mathrm{OMS}^{7}$, trata-se do tratamento em larga escala com o uso de pelo menos uma dose de penicilina Bezantina até a $24^{\mathrm{a}}$ semana de gestação. Entretanto, embora o quantitativo elevado nos índices de busca e realização pelo pré-natal, tal fato não correspondeu em uma redução de casos SC, por exemplo, evidenciando erros presentes durante esse acompanhamento, como a interpretação equivocada de resultados de exames característicos desse período, afetando a detecção de sífilis materna e de SC, promovendo um aumento da quantidade de diagnósticos apenas no momento do parto ${ }^{11,21}$.
Durante a verificação da taxa de mortalidade em menores de 1 ano para os casos de SC, é perceptível valores crescentes gradativos ao longo do intervalo determinado, atingindo o seu maior valor ao final do período analisado, de 0,08/1000 NV. Tal aumento da taxa de mortalidade por SC, também é descrito no Boletim Epidemiológico, com taxas de 2,8 por 100 mil NV no ano de 2001, alcançando 5,5 por 100 mil NV em 2013, o fim do período avaliado. Por fim, estudos apontam que SC é um fator impulsionador de casos relacionados à mortalidade perinatal e complicações gestacionais ${ }^{22-24}$.

Ademais, estudos em diversos contextos indicam falhas diretas durante o manejo de casos de sífilis materna que influenciam diretamente na quantidade elevada de casos de transmissão vertical, considerados evitáveis, quando devidamente diagnosticados e tratados ${ }^{25}$. Embora cerca de dois terços desse quantitativo sejam casos assintomáticos, existe um impacto da SC na viabilidade fetal destacada pela comparação entre a proporção de casos de óbitos fetais e as principais complicações presentes nesta doença, como a prematuridade e a restrição do crescimento intrauterino, com indicativos de um risco de dez vezes maior de mortalidade a partir da presença da sífilis materna ${ }^{26-27}$. Desse modo, como a SC é um fator sentinela para a descrição da qualidade de assistência pré-natal, tal cenário no país indica a necessidade de melhorias na infraestrutura das unidades de saúde brasileiras, capacitação dos agentes de saúde e uma demanda adequada desse grupo para um acompanhamento integral e adequado para grávidas portadoras de sífilis ${ }^{11}$.

É preciso salientar, também, os limites do presente estudo ao usufruir de dados secundários, tendo em vista questões intrínsecas a tal análise, como a subnotificação de casos, o preenchimento incompleto dos registros de neonatos e a existência de informações conflitantes ou ignoradas nos registros presentes na plataforma para a coleta de dados. Consequentemente, novas publicações devem ser incentivadas em torno da temática abordada, visto sua relevância, incidência e impacto significativo no contexto nacional.

Por fim, destaca-se a correlação positiva da incidência e da mortalidade por casos de SC entre menores de 1 ano no Brasil ao longo dos anos analisados, sendo perceptível elevados índices de ambas as variáveis entre as cincos regiões geográficas, em especial na região Sudeste, com $43,14 \%$ dos casos notificados, sugerindo a necessidade de maior atenção à triagem da doença no pré-natal e de melhorias na infraestrutura e na capacitação dos serviços de saúde voltados à redução da mortalidade infantil por tal enfermidade na população brasileira. 


\section{REFERÊNCIAS}

1. Singh $A E$, Romanowski B. Syphilis: review with emphasis on clinical, epidemiologic and some biologic features. Clin Microbiol Rev. 1999;12(2):187-209.

2. Avelleira JCR, Bottino G. Sífilis: diagnóstico, tratamento e controle. An Bras Dermatol. 2006;81(2):111-26.

3. Organização Mundial da Saúde. Eliminação mundial da sífilis congênita: fundamento lógico e estratégia para ação. Genebra: Organização Mundial da Saúde; 2008. 46 p.

4. Valderrama J, Zacarías F, Mazin R. Maternal syphilis and congenital syphilis in Latin America: big problem, simple solution. Rev Panam Salud Publica. 2004;16(3):211-7.

5. Deperthes BD, Meheus A, O'Reilly K, Broutet N. Maternal and congenital syphilis programmes: case studies in Bolivia, Kenya and South Africa. Bull World Health Organ. 2004;82(6):410-6.

6. Klausner JD. The sound of silence: missing the opportunity to save lives at birth. Bull World Health Organ. 2013;91(3):158.

7. World Health Organization. Investment case for eliminating mother-to-child transmission of syphilis: promoting better maternal and child health and stronger health systems. Geneva: World Health Organization; 2012. 30 p.

8. Gonzales MA. Regional initiative for the elimination of mother-to-child transmission of HIV and congenital syphilis in Latin America and the Caribbean: regional monitoring strategy [Internet]. Washington, DC: Pan American Health Organization; 2010 [citado 9 set 2020]. 65 p. Disponível em: https://iris.paho.org/bitstream/ handle/10665.2/9992/9789275131114_ eng.pdf?sequence $=1$ \&isAllowed $=y$

9. Brasil. Ministério da Saúde. Portaria $n^{\circ} 542$ de 22 de dezembro de 1986. Para efeitos de Aplicação da Lei $n^{\circ} 6.259$ de 30 de outubro de 1975, que dispõe sobre o Sistema Nacional de Vigilância Epidemiológica e dá outras providências, ficam incluídas na relação constante da Portaria Ministerial $n^{\circ} 608 \mathrm{Bsb}$, de 28 de outubro de 1979, a Síndrome da Imunodeficiência Adquirida Sida/Aids e a Sífilis Congênita.
Brasília: Diário Oficial da República Federativa do Brasil. 1986 Dez 24; Seção 1:19827.

10. Brasil. Ministério da Saúde. Secretaria de Vigilância em Saúde. Departamento Nacional de Vigilância Epidemiológica. Doenças infecciosas e parasitárias: guia de bolso [Internet]. Brasília: Ministério da Saúde; 2010 [citado 9 set 2020]. 444 p. Disponível em: https://bvsms.saude.gov.br/bvs/ publicacoes/doencas_infecciosas_ parasitaria_guia_bolso.pdf

11. De Lorenzi DRS, Madi JM. Sífilis Congênita como Indicador de Assistência Pré-natal. Rev Bras Ginecol Obstet. 2001; 23(10):647-52.

12. Malta DC, Duarte EC, Almeida MF, Dias MAS, Morais Neto OL, Moura L, et al. Lista de causas de mortes evitáveis por intervenções dos Sistema Único de Saúde do Brasil. Epidemiol Serv Saude. 2008;16(4):233-44.

13. Komka MR, Lago EG. Sífilis congênita: notificação e realidade. Sci Med. 2007; 17(4):205-11.

14. Brasil. Ministério da Saúde. Secretaria de Vigilância em Saúde. Departamento Nacional de DST, Aids e Hepatites Virais. Boletim Epidemiológico: sífilis. Brasília: Ministério da Saúde; 2012.

15. Freitas LV, Teles LMR, Lima TM, Vieira NFC, Barbosa RCM, Pinheiro $\mathrm{AKB}$, et al. Exame físico no pré-natal: construção e validação de hipermídia educativa para a Enfermagem. Acta Paul Enferm. 2012;25(4),581-88.

16. Instituto Brasileiro de Geografia e Estatística. Organização do território/estrutura territorial, áreas dos municípios [Internet]. 2019 [citado 9 set 2020]. Disponível em: https://www.ibge.gov.br/geociencias/ organizacao-do-territorio/estruturaterritorial/15761-areas-dosmunicipios. html?=\&t=o-que-e

17. Brasil. Ministério da Saúde. Secretaria de Vigilância em Saúde. Departamento Nacional de DST, Aids e Hepatites Virais. Boletim epidemiológico: sífilis. Brasília [Internet]. 2015 [citado 9 set 2020]. Disponível em: http://www.aids.gov.br/ pt-br/node/88
18. Domingues RMSM, Szwarcwald CL, Souza PRB Jr, Leal MC. Prevalence of syphilis in pregnancy and prenatal syphilis testing in Brazil: birth in Brazil study. Rev Saude Publica. 2014;48(5): 766-74.

19. Cavalcante PAM, Pereira RBL, Castro JGD. Sífilis gestacional e congênita em Palmas, Tocantins, 2007-2014. Epidemiol Serv Saude. 2017;26(2):255-64.

20. Read P, Fairley CK, Chow EPF. Increasing trends of syphilis among men who have sex with men in high income countries. Sex Health. 2015;12(2):155-63.

21. Magalhães DMS, Kawaguchi IAL, Dias A, Calderon IMP. Sífilis materna e congênita: ainda um desafio. Cad Saude Publica. 2013;29(6):1109-20.

22. Saraceni V, Guimarães MHFS, Theme Filha MMT, Leal MC. Mortalidade perinatal por sífilis congênita: indicador da qualidade da atenção à mulher e à criança. Cad Saude Publica. 2005;21(4):1244-50.

23. Nascimento MI, Cunha AA, Guimarães EV, Alvarez FS, Oliveira SRSM, Bôas ELV. Gestações complicadas por sífilis materna e óbito fetal. Rev Bras Ginecol Obstet. 2012;34(2):56-62. PubMed PMID: 22437763.

24. Azevedo AC, Drumond EF, Gonçalves RV, Machado CJ. Evolução da qualidade das informações das declarações de óbito com menções de sífilis congênita nos óbitos perinatais no Brasil. Cad Saude Colet. 2017;25(3):259-67.

25. Warner L, Rochat RW, Fichtner RR, Stoll BJ, Nathan L, Toomey KE. Missed opportunities for congenital syphilis prevention in an urban southeastern hospital. Sex Transm Dis. 2001;28(2):92-8.

26. Araújo EC, Moura EFA, Ramos FLP, Holanda VGDA. Sífilis congênita: incidência em recém-nascidos. $J$ Pediatr. 1999;75(2):119-25.

27. Aquino MM, Cecatti JG, Mariani Neto C. Risk factors associated to fetal death. Sao Paulo Med J. 1998;116(6):1852-7. 